\title{
Análise de Área de Cobertura de Dispositivo IoT para Monitoramento em Smart Farm
}

\author{
João Paulo Lemos Escola ${ }^{1,3}$, Rodrigo Capobianco Guido ${ }^{2}$, Ivan Nunes da Silva ${ }^{3}$, \\ Lucas Exposto Soares ${ }^{1,3}$ \\ jpescola@ifsp.edu.br; guido@ieee.org; nsilva@sc.usp.br; lucas.exposto@ifsp.edu.br \\ ${ }^{1}$ Instituto Federal de São Paulo (IFSP), 14781-502, Barretos-SP, Brasil \\ ${ }^{2}$ Instituto de Biociências, Letras e Ciências Exatas, Universidade Estadual Paulista (UNESP), 15054-0oo, \\ São José do Rio Preto-SP, Brasil \\ ${ }^{3}$ Escola de Engenharia de São Carlos, Universidade de São Paulo (USP), 13566-590, São Carlos-SP, Brasil
}

DOI: 10.17013/risti.42.1-11

Resumo: A melhoria do processo de monitoramento de insetos-praga em área agrícola é um desafio para os cientistas. A cigarra é um inseto-praga da lavoura de café que tem como característica marcante a emissão de som. Um dispositivo capaz de auxiliar o agricultor no manejo desses insetos pode ser de grande auxílio no manejo desses insetos. O presente trabalho busca aprimorar o processo de monitoramento automatizado de cigarras em lavoura de café analisando o alcance do dispositivo capaz de detetar a presença das cigarras no campo. Os resultados mostraram promissores, encorajando os pesquisadores na continuidade dos estudos.

Palavras-chave: Internet das Coisas; Agricultura 4.0; Cigarras.

\section{IoT Device Coverage Analysis for Smart Farm Monitoring}

\begin{abstract}
Improving the process of monitoring pest insects in agricultural areas is a challenge for scientists. The cicada is an insect-pest of the coffee crop that has a distinctive feature of sound emission. A device capable of assisting the farmer in the management of these insects can be of great help in the management of these insects. This work seeks to improve the process of automated monitoring of cicadas in coffee fields by analyzing the range of the device capable of detecting the presence of cicadas in the field. The results showed promising results, encouraging researchers to continue their studies.
\end{abstract}

Keywords: Internet of Things; Agriculture 4.0; Cicadas.

\section{Introdução}

De acordo com (MADAKAM, RAMASWAMY \& TRIPATHI, 2015), a Internet das Coisas (IoT) é uma tecnologia que está revolucionando a computação e as telecomunicações. Esse termo se refere aos diversos dispositivos (coisas), desde microcomputadores, 
passando por smartphones e microcontroladores, até geladeiras, lâmpadas ou roupas, que podem ser utilizados para as mais diversas aplicações, interligando-se ou fornecendo serviços por meio da rede mundial de computadores (Internet).

Dentre as aplicações possíveis com uso de IoT estão identificação, automação, monitoramento e controle. Para isso, dispositivos como microcontroladores e sensores são empregados para realizar as tarefas que utilizam a Internet para se interligar ou fornecer respostas aos usuários.

Quando utiliza-se IoT em aplicações de monitoramento, como em (DEZOTTI et al., 2019), (ZGANK, 2020) e (MITEV et al., 2020), que usam um módulo de microfone para captura de áudio, é desejável conhecer sua cobertura, ou seja, o alcance do dispositivo, a fim de realizar um levantamento do número de dispositivos necessários para monitorar determinada área e conhecer seu efetivo custo de implantação.

O objetivo deste trabalho é analisar o alcance de um sistema desenvolvido para monitoramento de lavoura de café, configurando-se como uma aplicação do tipo Smart Farm, que utiliza o paradigma IoT, com um microcontrolador integrado a sensores adicionais, sendo o principal deles o sensor de captura de áudio.

Este artigo está dividido da seguinte forma: à frente temos uma revisão da literatura, em seguida o capítulo "Metodologia" exprime os detalhes dos experimentos. No capítulo seguinte apresentam-se os resultados e, em seguida, as conclusões e trabalhos futuros.

\subsection{Internet das Coisas - IoT}

Há muitos anos os seres humanos sonham em viver em lugares modernos e futuristas, em que os recursos estão à disposição, tornando suas vidas mais fáceis e produtivas, onde problemas atuais são resolvidos pelas máquinas e robôs fazem o seu trabalho pesado, possibilitando uma vida de conforto e com tranquilidade.

Nesse contexto, a IoT permite atualmente aos profissionais criarem soluções que se comunicam pela Internet, executam tarefas automatizadas e entregam serviços às pessoas tanto virtualmente, quando o refrigerador faz um pedido de compra ao supermercado, quanto fisicamente, quando aciona o aspirador de pó para limpar o seu quarto. Recursos antes restritos aos sonhos hoje estão mais próximos de se tornar realidade.

Com o barateamento dos componentes eletrônicos e aumento da velocidade e abrangência da Internet, a IoT ganha força permitindo desenvolvimento de aplicações em vasta gama.

De acordo com (MADAKAM, RAMASWAMY \& TRIPATHI, 2015), essa tecnologia deverá transformar os objetos do mundo real em objetos virtuais inteligentes, unificando-os em uma infraestrutura comum, dando-nos o controle e mantendo-nos informados sobre o estado dos dispositivos.

Atualmente, os microcontroladores, como o Arduino (MCROBERTS, 2011; EVANS, NOBLE \& HOCHENBAUM, 2013) e ESP8266 (SCHWARTZ, 2016; OLIVEIRA, 2017), permitem modelar um código que personaliza seu comportamento, normalmente integrado a um ou mais módulos adicionais ou sensores, aumentando a gama de recursos e possibilidades do projeto. Dentre suas importantes funcionalidades estão a capacidade 
de processamento e memória, conexão com a Internet e bluetooth, tudo isso com baixo custo e reduzido consumo de energia.

Dentre os módulos adicionais está o sensor de áudio, como o MAX9814 (RAMIREZ \& MOUMTADI, 2018; RAHMAWATI, HARYANTO \& SAKARIYA, 2019; MALLIK, CHOWDHURY \& CHTTOPADHYAY, 2019), que possibilita criar aplicações que realizam processamento digital de sinais de áudio, permitindo a criação de aplicações antes possíveis apenas com Processadores Digitais de Sinais (DSPs) ou microcomputadores.

\subsection{Monitoramento de Áudio}

O monitoramento de áudio consiste em analisar o som em determinada situação por meio de processamento digital de sinais de áudio utilizando técnicas como Transformada Rápida de Fourier (FFT) (HAYKIN \& VEEN, 2001) ou Transformada Discreta Wavelet (DWT) (GUIDO, 2017).

Em (SANBORN, HEATH \& HEATH, 2009), tem-se um exemplo de estudo relacionado ao monitoramento de áudio, onde os sons emitidos por uma espécie de cigarra são analisados cuidadosamente após a captura em campo, em diversas regiões dos EUA, utilizando gravador e microfone Uher 4000. De acordo com os autores, o inseto emite uma alta frequência, o que se torna uma vantagem, pois seus predadores (pássaros e lagartos) não ouvem, resultando em baixa pressão predatória.

No artigo (LEE, 2008) apresenta-se uma pesquisa na qual é feito o levantamento das espécies de cigarras na península coreana. Os sons das cigarras são capturados e analisados por oscilogramas e sonogramas. Os dados são comparados, não para detetar, mas para diferenciar as espécies.

No trabalho publicado por (COLE, 2008), é realizada a captura de sons de cigarras com um microfone $\mathrm{C} 451 \mathrm{E}$ e um gravador DAT. A maioria das gravações aconteceu a $1 \mathrm{~m}$ ou mais de distância. As frequências foram analisadas por espectrogramas gerados a partir da FFT. Por fim é realizada a comparação entre duas espécies, analisando os gráficos dos sons dos machos.

No trabalho de (MITEV et al., 2020), utiliza-se IoT para monitoramento de áudio, na forma de um detetor de dispositivos espiões, onde os sons capturados por voz em ambiente Smart Home são monitorados a fim de filtrar outros dispositivos que enviam comandos de áudio para a Internet, não havendo relatos de mensuração do alcance ou cobertura do protótipo.

No artigo de (ZGANK, 2020) é realizado monitoramento de áudio em Smart Farm aplicado à apicultura, onde dispositivos IoT são utilizados para detetar o início do processo de enxameação, por meio de processamento digital de áudio combinado com técnicas como Modelos Ocultos de Markov (HMM) e Modelos de Mistura Gaussiano (GMM), entretanto não foi realizada uma análise de cobertura acústica do dispositivo.

\subsection{Smart Farm}

Muitos são os países que tem como base econômica a agricultura. De acordo com (GUANZIROLI, SABBATO \& BUAINAIN, 2020), 7.5\% do território brasileiro é composto 
por lavouras, perfazendo cerca de 351 milhões de hectares e a disponibilidade de terras agriculturáveis coloca o Brasil à frente de muitos países.

A evolução do trabalho no campo e os lucros crescentes possibilitam aumento do investimento em tecnologia, evidenciando sistemas que busquem melhorar os processos produtivos, aumentando a produção e consequentemente os lucros. Ainda segundo (GUANZIROLI, SABBATO \& BUAINAIN, 2020), em uma análise entre os anos de 2006 a 2017 constata-se aumento da escala produtiva e conteúdo tecnológico, indicando a diferença que as tecnologias fazem no aumento da produtividade com maior eficiência, gerenciamento dos processos de produção e de insumos modernos.

O conceito de Smart Farm consiste no aproveitamento da Tecnologia da Informação e Comunicação (TIC) com intuito de promover a eficiência, produtividade e lucratividade nos ambientes agrícolas (O'GRADY \& O'HARE, 2017; BARRIENTOS-AVENDAÑO et al., 2019). Esse paradigma está cada vez mais evoluindo junto com a evolução da IoT, pois este possibilita o desenvolvimento de aplicações de baixo custo e com importantes resultados, cada vez mais direcionados ao escopo de Smart Farm (MONTOYA et al., 2017).

Para (WARK et al., 2007) os problemas enfrentados pela agricultura como mudanças climáticas, falta de água e falta de mão de obra aumentam a preocupação da população com questões como segurança alimentar e impacto ambiental, podendo a Smart Farm ser importante aliada na busca de soluções para esses problemas.

Segundo (O’GRADY \& O’HARE, 2017) essas tecnologias são subutilizadas na agricultura, entretanto, podem prover aumento da produtividade por auxiliarem na tomada de decisões, tendo como exemplos a análise de alimentação de animais, monitoramento acústico, análise de área de plantações de algodão e de doenças comuns em gado leiteiro por meio de sensores não invasivos e de baixo custo.

No trabalho de (MUANGPRATHUB et al., 2019), um sistema IoT aplicado a Smart Farm foi desenvolvido utilizando sensores de umidade de solo, sensores de temperatura, uma aplicação para smartphone e uma aplicação Web buscando otimizar a produtividade e reduzir custos de produção. O sistema possibilita acionamento manual ou automático de válvulas solenoides para irrigação.

\subsection{Trabalhos correlatos}

No artigo de (SHAH, TARIQ \& LEE, 2018) IoT e áudio são empregados em um sistema de análise de segurança doméstica por meio do som capturado em ambiente residencial interno. Com auxílio da técnica de aprendizagem de máquinas (Machine Learning) o sistema envia à Internet trechos de sinais de áudio classificando-os dentro de categorias prévias, relacionadas à violência doméstica. Um conjunto de dispositivos é espalhado pela residência, com um dispositivo em cada cômodo e não houve análise da cobertura ou do alcance máximo de cada dispositivo.

O trabalho de (NEBHEN, FERREIRA \& MANSOURI, 2020) apresenta um amplificador de instrumentação de baixo ruído para uso em IoT, buscando maior sensibilidade com baixo consumo de energia. Nesse trabalho também não há uma análise do alcance do dispositivo, sendo os testes realizados em laboratório à uma distância de 1 metro do microfone. 
Em (ROCHESTER et al., 2019) um dispositivo IoT é utilizado para monitoramento do besouro-do-pinheiro a partir de seu ruído emitido. Também não é analisado o alcance do dispositivo apesar de utilizarem tecnologia de longo alcance para transmissão dos dados entre os dispositivos da lavoura e seu gateway.

No artigo de (ALEXANDRU et al., 2018) um dispositivo IoT é desenvolvido para captura de diversos tipos de dados, incluindo captura de áudio para mensurar poluição sonora. A implantação da rede de monitoramento considerou um raio de $1 \mathrm{Km}$ entre os dispositivos, considerando a capacidade de transmissão de dados e não de captura do sinal sonoro.

Nos trabalhos de (DEZOTTI et al., 2019; ESCOLA et al., 2020a, 2020b) um dispositivo é desenvolvido para captura de sinais acústicos de cigarras em lavouras de café, realizando o devido monitoramento da área de cobertura da rede de dispositivos. O presente artigo busca aperfeiçoar seus estudos analisando a área de cobertura, ou seja, a capacidade de alcance do dispositivo em relação ao sinal emitido pela cigarra a fim de estimar o número de dispositivos que será necessário para implantação em cada determinada área a ser coberta.

Não foram encontrados, na literatura, trabalhos com foco na análise de cobertura do sensor de áudio para dispositivos IoT, mostrando a importância do presente trabalho no auxílio de muitos trabalhos de pesquisas que aplicam Processamento Digital de Sinais (PDS) em IoT.

\section{Metodologia}

Utilizando um microcontrolador ESP32 (OLIVEIRA, 2017) foi desenvolvido um dispositivo, chamado INS (Figura 1), para monitoramento do sons emitidos pelas cigarras em lavouras de café, com intuito de realizar um monitoramento automatizado, contribuindo para a melhoria do processo, retirando a necessidade de uso de trabalho manual e melhorando sua qualidade (DEZOTTI et al., 2019; ESCOLA et al., 2020a, 2020b).

O foco do presente trabalho está na mensuração do alcance do microfone, integrado ao INS, em relação a uma ou mais cigarras possivelmente presentes na lavoura e assim conhecer quantos dispositivos são necessários para obter o melhor rendimento e cobertura de uma determinada área, sítio, fazenda ou lavoura específica.

O dispositivo INS, a exemplo de grande parte dos projetos IoT, é composto por um conjunto de módulos de expansão, também chamados de shields ou sensores, de baixo custo, que permitem incorporar recursos a um projeto IoT, ex: sensor de temperatura, sensor de presença, relês etc. O módulo MAX9814 permite a captura de som para processamento interno do dispositivo.

Para realização dos testes, além do INS foram utilizados um laptop com processador Intel(R) Core(TM) i7-480oMQ CPU @ 2.70GHz, 32GB de memória RAM e disco rígido SSHD de 1TB em ambiente Linux; um smartphone (emissor) com intuito de executar um arquivo de áudio simulando o som da cigarras; e um smartphone (decibelímetro) executando um aplicativo simulando um decibelímetro. $\mathrm{O}$ arquivo com som de cigarras 


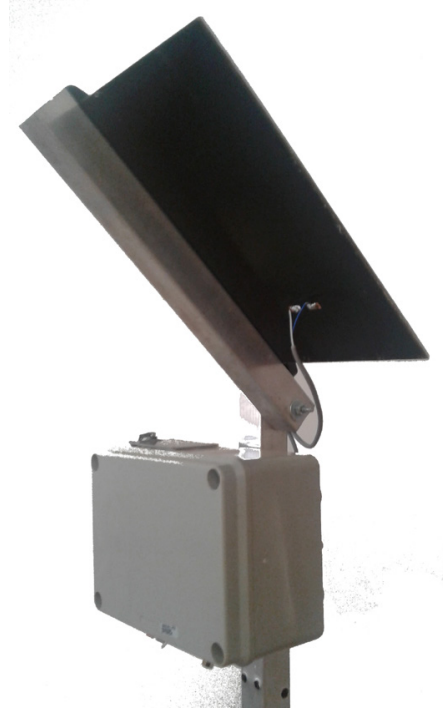

Figura 1 - Dispositivo INS

faz parte de um banco de dados de arquivos capturados em lavoura de café por um especialista pertencente ao grupo de pesquisa.

O dispositivo INS captura, de forma intermitente, 1200 milissegundos de áudio, armazenando os dados em um vetor, em seguida os dados são processados, as 25 energias resultantes de sub-bandas de frequência do sinal são apresentadas via saída padrão serial e capturadas pelo laptop. A Figura 2 ilustra o processo realizado durante os testes, onde $D$ é a distância entre o emissor e o INS. São capturados 13 sinais em cada teste, variando as distâncias $D$ entre 2 e 20 metros, conforme exposto adiante.

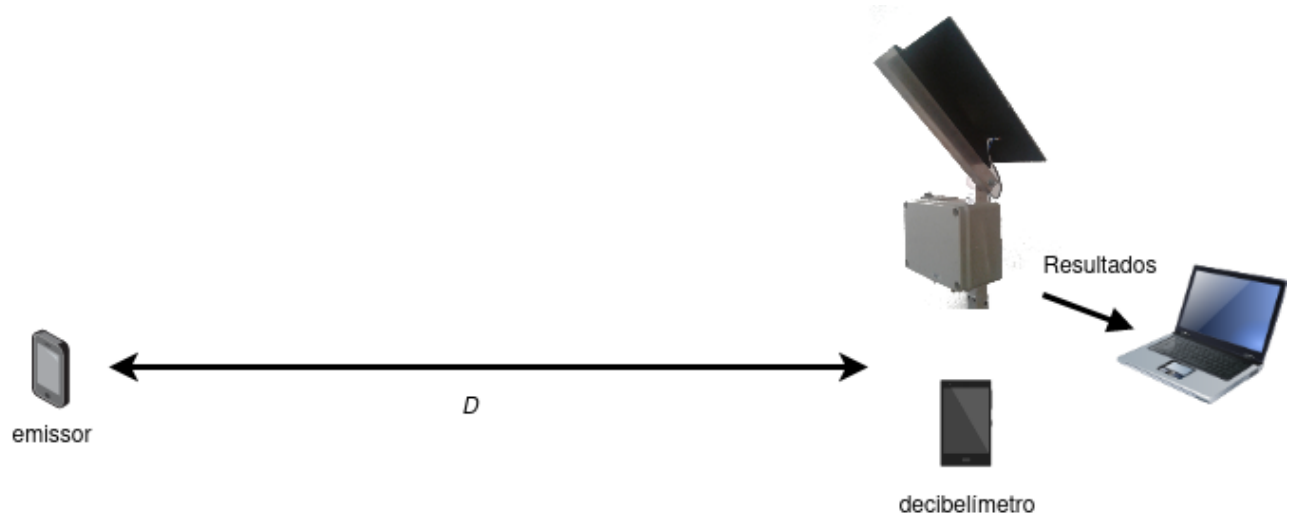

Figura 2 - Esquema utilizado nos testes 
De posse dos 13 sinais resultantes de cada teste, compostos de 25 energias cada, os sinais são submetidos a uma Máquina de Vetor de Suporte (SVM), previamente treinada, a fim de verificar sua acurácia. Além desses, as intensidades mínima, média e máxima do sinal, em decibéis, também são registradas.

\section{Resultados e Discussão}

Conforme descrito na metodologia, para a realização dos testes foram utilizados quatro dispositivos de hardware: dois smartphones (emissor e decibelímetro) e um laptop, além do dispositivo INS. Esse conjunto de dispositivos permitiu a organização de um aparato tecnológico com intuito de simular o ambiente da lavoura, a fim de testar devidamente a aplicabilidade do sistema.

O smartphone emissor foi posicionado à uma distância $D$ do INS, executando um arquivo de áudio WAV capturado previamente em lavoura de café por um membro especialista do grupo de pesquisa. $\mathrm{O}$ arquivo de áudio foi configurado para ser executado indefinidamente a fim de manter constante o som da cigarras emitido pelo equipamento.

O smartphone decibelímetro foi posicionado ao lado do INS para capturar a intensidade do sinal recebido durante cada teste. Os níveis de potência do sinal são importante parâmetro para analisar a efetiva intensidade de recepção do sinal pelo dispositivo INS, assim, esses dados foram incluídos na tabela de resultados apresentada adiante.

O laptop foi responsável por apresentar os resultados das capturas realizadas pelo dispositivo INS durante os testes, permitindo ao pesquisador monitorar o progresso das análises em tempo real. Ao final de cada teste, os resultados foram registrados em arquivo texto que permitiu posterior análise dos resultados, comparando-os entre cada conjunto de testes em diferentes distâncias.

No início de cada teste é aferida a distância entre o emissor e o INS com o auxílio de uma trena, em seguida são ativados o decibelímetro, o emissor e, finalmente, o dispositivo INS é ativado para dar-se início ao teste. Ao apresentar 13 resultados o teste é manualmente finalizado.

Cada um dos 13 sinais é armazenado no algoritmo em um vetor de X posições, que é processado através da Transformada Discreta Wavelet Packet (DWTP). Cada um dos 13 sinais de cada teste dispendeu, em média, 56522 milissegundos para ser capturado, processado e apresentado na tela.

Na Tabela 1 apresentam-se os resultados obtidos nos experimentos. Foram realizados 5 testes, sendo o primeiro sem emissão de sons de cigarras e os demais com som de cigarras, sendo o emissor foi posicionado a uma distância variando de $2 \mathrm{~m}$ a $20 \mathrm{~m}$. A fim de analisar a intensidade do sinal recebido pelo dispositivo INS em cada teste, foram incluídas na mesma tabela o valor da intensidade mínima, média e máxima, capturados pelo dispositivo decibelímetro. A acurácia resultante é apresentada de acordo com o referido teste.

Conforme se pode verificar na Tabela 1, no teste sem utilização do emissor, ou seja, sem incidência de cigarras, todos os 13 sinais foram considerados "ruído" pela SVM, perfazendo um total de $100 \%$ de acerto. Ressalta-se aqui a importância do resultado neste primeiro teste, que não apresentou falsos positivos, que poderiam debilitar o 


\begin{tabular}{lcccc}
\hline Distância $\boldsymbol{D}$ & dB min & dB min & dB min & Acurácia \\
\hline Sem cigarras & 59 & 60.0 & 82 & $100 \%$ \\
\hline $2 m$ & 73 & 76.4 & 83 & $84.6 \%$ \\
\hline $5 m$ & 40 & 73.3 & 80 & $84.6 \%$ \\
\hline $10 m$ & 44 & 64.1 & 79 & $100 \%$ \\
\hline $20 m$ & 39 & 55.4 & 81 & $100 \%$ \\
\hline
\end{tabular}

Tabela 1 - Bateria de testes com sons de cigarras.

sistema e até mesmo inviabilizá-lo, visto que o monitoramento da lavoura tem como um dos maiores pilares a real deteção de um número mínimo de indivíduos presentes para uma rápida tomada de decisões para aplicação de defensivos ou outro tipo de manejo por parte do agricultor.

Na Tabela 2 tem-se a matriz de confusão do segundo e terceiro testes, onde o emissor foi posicionado à uma distância de $2 \mathrm{~m}$ e de $5 \mathrm{~m}$ do INS. Dos 13 sinais testados, 11 foram corretamente classificados e dois sinais foram classificados como "ruído" incorretamente, resultando em uma acurácia de $84.6 \%$ em ambos os casos. Ressaltamos aqui a importância desse resultado, que se mostra promissor, mesmo com a incidência de falsos negativos (2 neste caso - 15.4\%), visto que, a importância do sistema está, inicialmente, na deteção dos indivíduos na lavoura. Assim sendo, os testes de $2 \mathrm{~m}$ e $5 \mathrm{~m}$ mostraram que o sistema pode falhar mas que ainda é eficiente para o problema a que se propõe.

\begin{tabular}{lcc}
\hline & Cigarra & Ruído \\
\hline Cigarra & 11 & 2 \\
\hline Ruído & 0 & 0 \\
\hline
\end{tabular}

Tabela 2 - Matriz de confusão dos testes com distância de 2m e 5m

Nos testes com distância de $10 \mathrm{~m}$ e de 20m, além do teste sem cigarras como visto anteriormente, todos os sinais foram corretamente classificados, apresentando 100\% de acurácia em cada um dos referidos testes, corroborando a hipótese de que o sistema pode se mostrando interessante recurso para monitoramento, considerando a distância empregada.

\section{Conclusões}

O presente trabalho conclui a primeira etapa de estudos relacionados à aferição da distância e alcance do dispositivo INS. Os testes, realizados em laboratório, não permitiram distâncias maiores que 20m devido às limitações de espaço físico, entretanto é desejável futuramente executar os testes em distâncias maiores em ambiente de lavoura de café a fim de analisar os resultados com outras variáveis como o vento.

Os testes sem cigarras foram importantes para verificar a existência de falsos positivos. O aproveitamento máximo apresentado nessa etapa mostrou que a SVM encontra-se 
satisfatoriamente treinada para o objetivo proposto no projeto, que é o monitoramento do inseto-praga cigarra em lavouras de café.

Em trabalhos futuros, deseja-se realizar as análises de desempenho da bateria e da placa solar, que fornecem alimentação ao dispositivo quando implantados em lavoura de café. Além desses, os testes com distâncias maiores em lavoura de café, a fim de obter resultados mais próximos aos reais em relação à implantação de um conjunto de INS em ambiente de produção, conhecendo os custos necessários para monitorar determinada área da plantação e realizar os demais testes para validação do sistema, como alcance de comunicação de dados entre os nós.

\section{Referências}

Alexandru, P., Andrei, M., Cristina-Madalina, S., \& Stan, O. (2018). Smart environmental monitoring beacon. En 2018 ieee international conference on automation, quality and testing, robotics (aqtr) (pp. 1-4). https://doi.org/10.1109/aqtr.2018.8402701

Barrientos-Avendaño, E., Rico-Bautista, D., Coronel-Rojas, L. A., \& Cuesta-Quintero, F. R. (2019). Granja inteligente: Definición de infraestructura basada en internet de las cosas, IpV6 y redes definidas por software. RISTI - Revista Ibérica de Sistemas e Tecnologias de Informação, (E17), 183-197.

Cole, J. A. (2008). A new cryptic species of cicada resembling tibicen dorsatus revealed bycalling song (hemiptera: Auchenorrhyncha: Cicadidae). Annals of the Entomological Society of America, 101(5), 815-823. https://doi.org/10.1093/ aesa/101.5.815

de Oliveira, S. (2017). Internet das coisas com esp8266, arduino e raspberry pi. Novatec Editora.

Dezotti, A. K., Cardoso, A. M., Soares, L. E., Maccagnan, D. H. B. (2019). Dispositivo de monitoramento de densidade populacional de insetos a partir de sinais acústicos emitidos. Brazilian Journal of Animal and Environmental Research, 2(5), 1781-1785.

Escola, J. P. L., Guido, R. C., Cardoso, A. M., Maccagnan, D. H. B., Ribeiro, J. M., \& Cardoso, J. R. F. (2020). A case study of wavelets and svm application in coffee agriculture: Detecting cicadas based on their acoustic and image patterns. En I. N. da Silva \& R. A. Flauzino (Eds.), Application of expert systems. Rijeka: IntechOpen. https//doi.org/10.5772/intechopen.90156

Escola, J. P. L., Guido, R. C., da Silva, I. N., Cardoso, A. M., Maccagnan, D. H. B., \& Dezotti, A. K. (2020). Automated acoustic detection of a cicadid pest in coffee plantations. Computers and Electronics in Agriculture,169, 105215. https://doi.org/10.1016/j.compag.2020.105215

Evans, M., Noble, J., \& Hochenbaum, J. (2013). Arduino em ação. Novatec Editora.

Guanziroli, C. E., Di Sabbato, A., \& Buainain, A. M. (2012). Evolução da agricultura familiar no Brasil (1996-2017). Uma Jornada Pelos Contrastes do Brasil, 191. https://doi.org/10.1590/s0103-20032012000200009 
Guido, R. C. (2017). Effectively interpreting discrete wavelet transformed signals. IEEE Signal Processing Magazine, 34(3), 89-100. https://doi.org/10.1109/ msp.2017.2672759

Haykin, S. S., \& Van Veen, B. (2001). Sinais e sistemas. Bookman.

Lee, Y. J. (2008). Revised synonymic list of cicadidae (insecta: Hemiptera) from the korean peninsula, with the description of a new species and some taxonomic remarks. Proceedings of the Biological Society of Washington, 121(4), 445-467. https://doi.org/10.2988/08-09.1

Madakam, S., Ramaswamy, R., \& Tripathi, S. (2015). Internet of things (iot): A literature review. Journal of Computer and Communications, 3(05), 164. https://doi.org/10.4236/jcc.2015.35021

Mallik, S., Chowdhury, D., \& Chttopadhyay, M. (2019). Development and performance analysis of a low-cost mems microphone-based hearing aid with three different audio amplifiers. Innovations in Systems and Software Engineering, 15(1), 17-25. https://doi.org/10.1007/s11334-019-00325-7

McRoberts, M. (2011). Beginning arduino. Apress.

Mitev, R., Pazii, A., Miettinen, M., Enck, W., \& Sadeghi, A.-R. (2020). Leakypick: Iot audio spy detector. En Annual computer security applications conference (pp. 694-705). https://doi.org/10.1145/3427228.3427277

Montoya, E. A. Q., Colorado, S. F. J., Muñoz, W. Y. C., \& Golondrino, G. E. C. (2017). Propuesta de una arquitectura para agricultura de precisión soportada en IoT. RISTI - Revista Ibérica de Sistemas e Tecnologias de Informação, (24), 39-56. https://doi.org/10.17013/risti.24.39-56

Muangprathub, J., Boonnam, N., Kajornkasirat, S., Lekbangpong, N., Wanichsombat, A., \& Nillaor, P. (2019). Iot and agriculture data analysis for Smart Farm. Computers and electronics in agriculture, 156, 467-474. https://doi.org/10.1016/j. compag.2018.12.011

Nebhen, J., Ferreira, P. M., \& Mansouri, S. (2020). A chopper stabilization audio instrumentation amplifier for iot applications. Journal of Low Power Electronics and Applications, 10(2), 13. https://doi.org/10.3390/jlpea10020013

O’Grady,M.J.,\&O'Hare,G.M.(2017).ModellingtheSmartFarm.Informationprocessing in agriculture, 4(3), 179-187. https://doi.org/10.1016/j.inpa.2017.05.001

Rahmawati, D., Haryanto, H., \& Sakariya, F. (2019). The design of coconut maturity prediction device with acoustic frequency detection using naive bayes method based microcontroller. JEEMECS (Journal Electr. Eng. Mechatron. Comput. Sci.). https://doi.org/10.26905/jeemecs.v2i1.2806

Ramírez, A. C., \& Moumtadi, F. (2018). Design of a hearing auxiliary for bilateral hypoacusia. International Journal of Engineering Sciences \& Research Technology. https://doi.org/10.5281/zenodo.1165570 
Rochester, E., Ma, J., Lee, B., \& Ghaderi, M. (2019). Mountain pine beetle monitoring with iot. En 2019 IEEE 5th world forum on internet of things (wf-iot) (pp. 513-518). http://dx.doi.org/10.1109/WF-IoT.2019.8767291

Sanborn, A., F., Heath, J. E., \& Heath, M. S. (2009). Long-range sound distribution and the calling song of the cicada beameria venosa (uhler) (hemiptera: Cicadidae). The Southwestern Naturalist, 54(1), 24-30. http://dx.doi.org/10.1894/JC-23.1

Schwartz, M. (2016). Internet of things with esp8266. Packt Publishing Ltd.

Shah, S. K., Tariq, Z., \& Lee, Y. (2018). Audio iot analytics for home automation safety. En 2018 IEEE international conference on big data (big data) (pp. 5181-5186). https://doi.org/10.1109/BigData.2018.8622587

Wark, T., Corke, P., Sikka, P., Klingbeil, L., Guo, Y., Crossman, C., . . Bishop-Hurley, G. (2007). Transforming agriculture through pervasive wireless sensor networks. IEEE Pervasive Computing, 6(2), 50-57. https://doi.org/10.1109/MPRV.2007.47

Zgank, A. (2020). Bee swarm activity acoustic classification for an iot-based farm service. Sensors, 20(1), 21. https://doi.org/10.3390/s20010021 